\title{
Early life stress and psychiatric disorder modulate cortical responses to affective stimuli
}

\author{
KATJA WEBER,${ }^{\mathrm{a}}$ GREGORY A. MILLER, ${ }^{\mathrm{b}}$ HARALD T. SCHUPP, ${ }^{\mathrm{a}}$ JENS BORGELT, ${ }^{\mathrm{a}}$ \\ BARBARA AWISZUS, ${ }^{\mathrm{a}}$ TZVETAN POPOV,${ }^{\mathrm{a}}$ THOMAS ELBERT, ${ }^{\mathrm{a}}$ AND BRIGITTE ROCKSTROH ${ }^{\mathrm{a}}$ \\ ${ }^{a}$ Department of Psychology, University of Konstanz, Konstanz, Germany \\ ${ }^{\mathrm{b}}$ Departments of Psychology and Psychiatry and Beckman Institute Biomedical Imaging Center, University of Illinois at Urbana Champaign, Urbana, \\ Illinois, USA
}

\begin{abstract}
Altered affective processing has been proposed as mediating between early life stress (ELS) and subsequent psycho pathology. The present study examined whether ELS influences affective cortical processing differently in psychiatric patients and healthy subjects. The number of stressful experiences before onset of puberty was assessed in 50 inpatients with diagnoses of Major Depressive Disorder, schizophrenia, drug addiction, or Borderline Personality Disorder and in 20 healthy comparison subjects. Subjects monitored pleasant, neutral, and unpleasant pictures during magneto encephalographic recording. Suppression of right posterior activity $160210 \mathrm{~ms}$ after stimulus onset was associated with certain diagnoses and high ELS. Results confirmed specific contributions of ELS versus adult stress, comorbid posttraumatic stress disorder, or depression.
\end{abstract}

Descriptors: Early life stress, Psychopathology, Affective modulation, IAPS, MEG

Visual stimuli with emotional content are frequent and effective prompts for cortical processing, including output systems mo tivating approach avoidance behavior (Lang, Bradley, \& Cuth bert, 1998a, 1998b). The modulation of cortical processing by the salience and valence of affective pictures has been verified by neuroimaging methods such as electroencephalography (EEG), magnetoencephalography (MEG), and functional magnetic res onance imaging (fMRI; e.g., Bradley et al., 2003; Junghöfer, Bradley, Elbert, \& Lang, 2001; Junghöfer, Schupp, Stark, \& Vaitl, 2005, Junghöfer et al., 2006; Sabatinelli, Bradley, Fitzsim mons, \& Lang, 2005; Schupp, Junghöfer, Weike, \& Hamm, 2003, 2004). Event related brain potential (ERP) studies consis tently reveal that the processing of pleasant and unpleasant stimuli is associated with an enhanced posterior negativity around $120300 \mathrm{~ms}$ poststimulus (early posterior negativity, EPN) compared to neutral stimuli (Junghöfer et al., 2001; Schupp et al., 2003). A recent event related magnetic field (ERF) study showed a strong and reliable magnetic counterpart to the EPN reported in ERP studies. Source analyses indicated strongly amplified processing of pleasant and unpleasant emotional pic tures compared to neutral material in occipito parieto temporal

Research was supported by the Deutsche Forschungsgemeinschaft (FOR 751). G. A. Miller was a guest professor at the University of Konstanz during a portion of this work. We are particularly grateful to $\mathrm{H}$. Watzl, K. Propster, K. Schonauer, S. Karr, and K. Hoffmann for di agnoses and to U. Lommen, N. Brunnemann, and M. Zeiller for assis tance in MEG recording and data preprocessing.

Address reprint requests to: Prof. Dr. Brigitte Rockstroh, Depart ment of Psychology, University of Konstanz, P.O.Box D23, D 78457 Konstanz, Germany. E mail: Brigitte.rockstroh@uni konstanz.de brain regions associated with visual processing (Peyk, Schupp, \& Elbert, 2008). According to a bivariate motivational model of emotion (Bradley, Codispoti, Cuthbert, \& Lang, 2001; Lang, Bradley, \& Cuthbert, 1997), enhanced attention to emotional cues supports the organization of efficient actions (serving appetitive and defensive goals) in response to events that can sustain or threaten the life of the organism.

Normal affective processing may be modified by individual factors, among them psychopathology or early life stress (ELS), purportedly through effects on neuronal and neuroendocrine sys tems that are also involved in affect regulation (e.g., Charmandari, Kino, \& Souvatzoglou, 2003). Many human studies have demon strated the impact of adverse or traumatic experiences on adult psychopathology (e.g., Heim \& Nemeroff, 2002; McEwen, 2003; Nemeroff, 2004) and on risk for it, such as depression (Heim, Plotsky, \& Nemeroff, 2004), schizophrenia (Thompson, Pogue Geile, \& Grace, 2004), personality disorder (Goodman, New, \& Siever, 2004), substance abuse (de Bellis, 2002; Sinha, 2005), and anxiety disorders, including posttraumatic stress disorder (PTSD; Scheller Gilkey, Moynes, Cooper, Kant, \& Miller, 2004; van der Kolk, Roth, Pelcovitz, Sunday, \& Spinazzola, 2005). Although ELS induced alterations in affective processing, neuroendocrine systems, and brain development are consistently reported as bio logical contributions to adult psychopathology, ELS may not be a differential risk factor for specific psychiatric disorders. Rather, ELS during sensitive periods of brain development is proposed to interact with genetic and/or pre or postnatal factors to influence broad vulnerability for disorder (Heim et al., 2004; Kendler, Kuhn, Vittum, Prescott, \& Riley, 2005; Maynard, Sikich, Lieberman, \& LaMantia, 2001). 
The impact of ELS on affective processing was exemplified in a study of 90 individuals without psychiatric diagnoses (Pole et al., 2007): The 25 subjects who reported childhood trauma also reported low positive emotion and exhibited large autonomic responses to threatening experimental stimuli compared to sub jects without ELS. Similarly, Cohen et al. (2006) found adverse childhood events to be associated with depression and anxiety in over 1,500 adults without psychiatric diagnoses. A behavioral state of despair or helplessness consequent to prenatal stress or lasting, inescapable stressors (Morley et al., 2003; Porsolt, Ber tin, \& Jalfre, 1978) has been documented in animal studies and related to neuroendocrine alterations (Cabib \& Puglisi Allegra, 1996). Lang, McTeague, and Cuthbert (2007) interpreted distress and negative affect as reflecting diminished activity of the defense system in anxious and depressive patients. They noted that de fense and reward systems overlap with the function of the stress system.

In summary, studies of ELS, affective processing, psychopa thology, and brain mechanisms have established many pairwise relationships. These various phenomena no doubt combine in complex, nonadditive ways. Childhood trauma may convey risk for adult psychopathology by altering emotional responses to subsequent stressors, for example. The present study explored whether retrospectively reported ELS is associated with differ ential cortical affective processing in patients with psychiatric disorders and healthy comparison subjects and whether this var ies by specific psychiatric disorder or by emotional stimulus fea tures. In particular, it examined attention capture by emotionally significant pictures as a function of ELS and diagnosis. Whole head MEG probed spontaneous, involuntary attention capture by emotional cues, which has been manifested in many previous studies as augmented activation over occipito parieto temporal regions from approximately 120 to $300 \mathrm{~ms}$ after stimulus onset. If ELS exerts long lasting effects on stress relevant systems, and if these influence cortical systems involved in affect processing and regulation (Bremner, 2002; Mayberg, 2003), high ELS may abolish or attenuate the early preferential processing of emo tional cues. Hence, the prediction was reduced responses to affective stimuli and reduced modulation of cortical activity by the affective valence of stimuli in the EPN time window.

Affective modulation has also been reported for activity around $100 \mathrm{~ms}$ after stimulus onset (e.g., Borgelt, Odenwald, Ruf, Elbert, \& Kissler, 2009; Junghöfer et al., 2003; Rockstroh, Junghöfer, Elbert, Buodo, \& Miller, 2006; Smith, Cacioppo, Larsen, \& Chartrand, 2003). Modulation of this effect by stress/ trauma or psychopathology varies across studies in that aug mented early activity relative to normal was reported for patients with PTSD (Borgelt et al., 2009; Junghöfer et al., 2003), and suppression of early activity relative to normal was reported for schizophrenia patients (Rockstroh et al., 2006). Therefore, the present study extended the search for possible effects of ELS or diagnosis to a second, earlier time window around $100 \mathrm{~ms}$. At issue was the temporal specificity of ELS effects on affective processing.

\section{Methods}

\section{Participants}

Table 1 summarizes demographic and clinical data for 50 inpa tients at the local Center for Psychiatry and 20 healthy compar ison individuals. Patients were recruited from several wards within the center and identified by the treating psychiatrists as meeting the inclusion criteria. Patients were included if they met International Classification of Diseases, 10th Revision (ICD 10) diagnoses of Major Depressive Disorder (MDD; F31 33), schizophrenia spectrum (F20, F25), drug addiction (DA; F19, F10), or Borderline Personality Disorder (BPD; F60.31) and if they were in a sufficiently remitted state to allow data collection (interview on life stress history and MEG protocol). As the cli entele of the center mainly includes long term inpatients, most were not in an acute state with severe symptoms. (This was con firmed by a global rating of symptom severity provided below.)

Table 1. Demographic and Clinical Data

\begin{tabular}{|c|c|c|c|c|c|c|c|}
\hline Group: $N$ & $\begin{array}{c}\text { Gender } \\
\text { (female/male) }\end{array}$ & $\begin{array}{c}\text { Age } \\
(M \pm S D)\end{array}$ & $\begin{array}{c}\text { Years } \\
\text { education }\end{array}$ & $\begin{array}{c}\mathrm{BDI} \\
(M \pm S D)\end{array}$ & $\begin{array}{c}\text { BPRS } \\
(M \pm S D)\end{array}$ & Medication $^{\mathrm{a}}$ & $\begin{array}{c}\text { Secondary ICD } \\
\text { diagnoses }\end{array}$ \\
\hline Patients: 50 & $17 / 33$ & $38.8 \pm 12.5$ & $12.2 \pm 2.9$ & $17.9 \pm 11.0$ & $52.1 \pm 9.0$ & & \\
\hline MDD: 19 & $10 / 9$ & $48.7 \pm 7.4$ & $12.5 \pm 3.0$ & $24.6 \pm 10.5$ & $51.1 \pm 7.9$ & $\begin{array}{l}\text { Mix: 7, TCA: 1, SSRI: } \\
\text { 10, AD mix: } 1\end{array}$ & $\begin{array}{l}\text { F4: } 2 \\
\text { F10.2: } 1 \\
\text { F6: dep: } 2 \\
\text { other: } 1 \\
\text { paranoid: } 1 \\
\text { schizoid: } 1 \\
\text { F43.2: } 2 \\
\text { None: } 9\end{array}$ \\
\hline Schizophrenia: 15 & $3 / 12$ & $34.5 \pm 10.7$ & $13.3 \pm 3.6$ & $13.6 \pm 9.9$ & $48.2 \pm 5.7$ & $\begin{array}{l}\text { Mix: } 8 \text {, } \\
\text { AD mix: } 1 \text {, atyp: } 6\end{array}$ & $\begin{array}{l}\text { F12: } 5 \\
\text { F33: } 1 ; 20.4: 2 \\
\text { None: } 7\end{array}$ \\
\hline Drug addiction: 10 & $0 / 10$ & $32.3 \pm 7.2$ & $10.6 \pm 1.4$ & $10.8 \pm 7.5$ & $59.5 \pm 10.4$ & None: 10 & $\begin{array}{l}\text { F60.3: } 1 \\
\text { F61.0: } 2 \\
\text { F12.1: } 1 \\
\text { None: } 6\end{array}$ \\
\hline $\begin{array}{l}\text { Borderline } \\
\text { Personality Disorder: } 6\end{array}$ & $4 / 2$ & $28.5 \pm 9.4$ & $11.2 \pm 1.5$ & $19.0 \pm 9.0$ & $50.4 \pm 11.8$ & $\begin{array}{l}\text { None: } 5 \text {, } \\
\text { Mix: } 1\end{array}$ & $\begin{array}{l}\text { F10: } 2 \\
\text { F65.4: } 1 \\
\text { F53: } 1 \\
\text { None: } 2\end{array}$ \\
\hline Comparison subjects: 20 & $8 / 12$ & $40.5 \pm 15.2$ & $15.3 \pm 2.9$ & $3.7 \pm 4.3$ & & None: 20 & \\
\hline
\end{tabular}

${ }^{\mathrm{a}} \mathrm{Mix}$ : combination of antidepressants (AD) and neuroleptics (N); TCA: tricylcic AD; SSRI: selective serotonin reuptake inhibitors; AD mix: com bination of TCA and SSRI; typ: typical neuroleptics; atyp: atypical neuroleptics; $\mathrm{N}$ mix: combination of typical and atypical neuroleptics; none: no medication. 
Patients with neurological conditions, head trauma with loss of consciousness, or intellectual disability were excluded.

For analyses comparing subgroups of inpatients by diagnosis, the primary diagnosis determined the subgroup. (Secondary di agnoses, reported in Table 1, were not fully assessed for some patients.) The four diagnostic subgroups differed in gender dis tribution, $\chi^{2}(3)=12.25, p<.01$, due to more male participants in the schizophrenia and DA subgroups versus balanced MDD and BPD subgroups, and in age, $F(3,46)=10.98, p<.001$, MDD patients being older than the other subgroups, who did not differ (post hoc $t$ tests evaluated significant effects as needed through out this study). Patient subgroups did not differ in years of ed ucation, $F(3,46)=2.2, p=.10$.

Severity of disorder was evaluated via the Brief Psychiatric Rating Scale (BPRS; Lukoff, Liberman, \& Nuechterlein, 1986) and the Beck Depression Inventory (German version; Hau tzinger, Bailer, Worall, \& Keller, 1994). MDD and DA sub groups exhibited higher BPRS scores than did the other two subgroups, $F(3,42)=3.83, p=.02$, and BDI scores were higher in patients with MDD than in the other subgroups, $F(3,46)=5.86, p=.002$, who did not differ. Except for partic ipants with drug addiction, most patients were on medication (see Table 1), the majority receiving combinations either of an tidepressants and neuroleptics, typical and atypical neuroleptics, or tricyclic and SSRI antidepressants. Monotherapy was rare.

Healthy volunteers were recruited from hospital staff, stu dents, and colleagues and were screened to be comparable to the patient sample in age, gender, and education. They were included if they did not meet criteria for a lifetime diagnosis (screened with the MINI interview; Ackenheil, Stotz Ingenlath, Dietz Bauer, \& Vossen, 1998), did not report any history of head trauma with loss of consciousness, and were free of psychoactive medication. The patient group and the comparison group were similar in gender balance and age (Fisher's exact test $p=.8$ and $p>.6$, respectively). Comparison subjects had more years of education than did patients, $F(1,68)=15.87, p<.001$.

Handedness was determined using the Edinburgh Handed ness Questionnaire (Oldfield, 1970). Right handedness was con firmed for 43 patients and 16 comparison subjects. Four patients and 2 comparison subjects were ambidextrous, and 3 patients and 2 comparison subjects were left handed. All participants had normal or corrected to normal vision. The study protocol was approved by the local ethics committee. All subjects gave written informed consent.

\section{Design and Procedure}

Prior to the MEG session, prepubertal and adult stress history was assessed with the German version of the Early Trauma In ventory (ETI; Bremner, Vermetten, \& Mazure, 2000; Heim, 2000). ${ }^{1}$ Stress load was defined as the number of reported events across four domains (emotional neglect, punishment, sexual abuse, other traumatic events), separately for (a) the time before the individual onset of puberty (taken as a measure of ELS) and (b) the time between 18 years and the assessment (labeled adult

${ }^{1}$ Psychometric properties have been confirmed for the ETI (Bremner et al., 2000; Bremner, Bolus, \& Mayer, 2007), the Traumatic Antecedent Questionnaire (TAQ; van der Kolk et al., 2005), and the Childhood Trauma Questionnaire (CTQ; Paivio \& Cramer, 2004). A retest of TAQ self reports after 36 months showed reliability of .8 (Garieballa et al., 2006). The high correlation between stressful events assessed by the ETI and traumatic events assessed by the PDS supports confidence in the retrospective self report of life events. stress, AS). Thus, ELS and AS were determined for each par ticipant. In addition, PTSD was diagnosed in an interview using the Posttraumatic Stress Diagnostic Scale (PDS; Foa, 1995).

Affective modulation was evaluated via picture ratings and electromagnetic cortical responses. Affective stimuli comprised 300 colored photographs from the International Affective Pic ture System IAPS (Center for the Study of Emotion and Atten tion, 2004): 150 stimuli were high arousal, 75 pleasant and 75 unpleasant valence, according to normative valence and arousal ratings (Lang, Bradley, \& Cuthbert, 1999), and 150 were low arousal, neutral valence stimuli. All pictures involved social scenes and were matched for size, contrast, and brightness. Pleasant and unpleasant pictures did not differ in rated arousal. As expected, both had significantly higher arousal scores than the neutral category. ${ }^{2}$

In a passive viewing task, pictures were presented for $660 \mathrm{~ms}$ with an offset to onset interstimulus interval of 700 to $900 \mathrm{~ms}$. Each picture was presented once within each of two series of 300 pictures (600 trials in total). Pleasant, neutral, and unpleas ant pictures were presented in random order. Within each affec tive category no more than two repetitions of the same picture category were allowed. Timing and sequence of stimulus pre sentation were controlled using Presentation software (Neuro behavioral Systems, Albany, CA). Participants were instructed that they would see a series of pictures with different content and that they should attend to each picture and try to avoid head and eye movements during each run, which lasted for about $15 \mathrm{~min}$.

After the MEG recording, each participant was asked to rate the valence and emotional arousal of 75 representative IAPS pic tures, 25 from each category. These pictures were randomly taken from the set of pictures presented during MEG recording. Picture ratings during MEG recording would have introduced an explicit evaluative task context and increased artifacts due to rating by button press. Therefore, ratings were collected following MEG recording relying on representative subsets of the pictures. The selected subsets of pleasant and unpleasant pictures were matched on arousal based on IAPS norms (Center for the Study of Emo tion and Attention, 2004). Pictures were presented without time limit in randomized order, and ratings were obtained with a com puterized version of the SAM (Bradley \& Lang, 1994). Arousal and valence of each picture were evaluated on a 9 point scale, with higher numbers indicating evaluation as more pleasant or more arousing. Ratings were not available for 9 patients.

\section{Data Acquisition and Analysis}

MEG was recorded while subjects were in a prone position, using a 148 channel magnetometer (Magnes 2500 WH, 4D Neuro imaging, San Diego, CA). Neuromagnetic data were continu ously recorded with a sampling rate of $678.17 \mathrm{~Hz}$ and a bandpass filter of 0.1 to $100 \mathrm{~Hz}$. For artifact control, the vertical and horizontal electrooculogram (EOG; recorded from four elec trodes placed near the left and right temporal canthus and above and below the right eye) and the electrocardiogram (ECG; from two electrodes attached to the right and left forearm) were re corded using a SynAmps amplifier (Neuroscan Laboratories,

${ }^{2}$ Because the IAPS set provided only 135 appropriate low arousal neutral slides, 15 pictures were added from picture databases on the Internet. The selection of pictures thematically similar to the ones of the IAPS set was validated by ratings of 30 student subjects using the Self Assessment Manikin (SAM; Bradley \& Lang, 1994) to match the nor mative ratings of IAPS pictures. 
Sterling, VA). The subject's nasion, left and right ear canal, and head shape were digitized with a Polhemus 3Space Fasttrack prior to each session. ${ }^{3}$

Following noise reduction, MEG data were corrected for heartbeat related artifacts: In time segments with $\mathrm{R}$ wave arti fact, an average magnetocardiogram was subtracted, calculated as a moving average over 20 heartbeats (4D Neuroimaging "cardiac comber" software). In addition, trials with eyeblinks were excluded from further data analyses. On average, $382 \mathrm{ar}$ tifact free trials per subject were available for further analysis, with no differences in number of trials between patients and comparison subjects for stimulus categories (pleasant, unpleas ant, neutral, all $F<1$ ). For artifact free trials, data epochs in cluding $100 \mathrm{~ms}$ before (baseline) and $660 \mathrm{~ms}$ after stimulus onset were averaged and filtered with a $1 \mathrm{~Hz}(12 \mathrm{~dB} /$ octave, forward shift) high pass filter and a $40 \mathrm{~Hz}(24 \mathrm{~dB} /$ octave, zero phase shift) low pass filter. BESA software (Megis Software GmbH, Munich, Germany) was used for preprocessing.

As in previous research, the L2 Minimum Norm Pseudoin verse (L2MNP) was used for inverse modeling, providing min imum norm estimates (MNE) of cortical activity without any assumption regarding the location and/or number of current sources (Baillet, Mosher, \& Leahy, 2001; Hamalainen \& Il moniemi, 1994; Hauk, 2004; Hauk, Keil, Elbert, \& Müller, 2002). Relying on EMEGS software (Junghöfer \& Peyk, 2004; see www.emegs.org), a spherical shell with 2 (azimuthal and polar direction) $\times 350$ evenly distributed dipoles served as the source model. A source shell radius of $87 \%$ of the individually fitted head radius was chosen, roughly corresponding to gray matter. A Tikhonov regularization parameter of 0.2 was applied. Magnetic field strength (independent of dipole direction) was calculated as the $3 \mathrm{D}$ vector length of the generator activity at each position for each subject, condition, and time point based on the averaged magnetic field distributions and the individual sensor positions.

In the source analysis, point wise analyses of variance (ANO VAs) were calculated separately for each dipole for identification of spatiotemporal modulation as a function of emotion. To avoid false positives, significant effects were considered only when they included a minimum of 21 continuous data points $(32 \mathrm{~ms})$ and when two adjacent representative dipoles showed emotion effects. This procedure determined a time window of differential brain activity $160210 \mathrm{~ms}$ after stimulus onset for left and right occipito parieto temporal regions of interest (ROI) that were scored for average activity among 21 dipoles (Figure 1). As MEG activity in this ROI seems comparable to the early posterior negativity (EPN) described in EEG studies (Peyk et al., 2008), it will be labeled as EPN from here on for the sake of simplicity. Employing the same procedure of point wise ANOVAs, differential activity in the in terval $80115 \mathrm{~ms}$ after stimulus onset was used to define a ROI scored from the average of 18 dipoles each in the left and right anterior cortex (Figure 1). Referring to the time window, this component was related to a M100.

\footnotetext{
${ }^{3}$ The nasion and the left and right ear canal served as index points and were used to define a right handed coordinate system, called the head frame coordinate system. The positive pole of the $x$ axis points to the front, the $y$ axis to the left, and the $z$ axis to the top of the head. The head shape information is used in standard analysis software for localization of activity sources (4D Neuroimaging WHS 1.2.6) by fitting a local sphere to the head shape underneath selected sets of adjacent channels. The subject's head position relative to the pickup coils of the MEG dewar was estimated before and after each session using these index points.
}
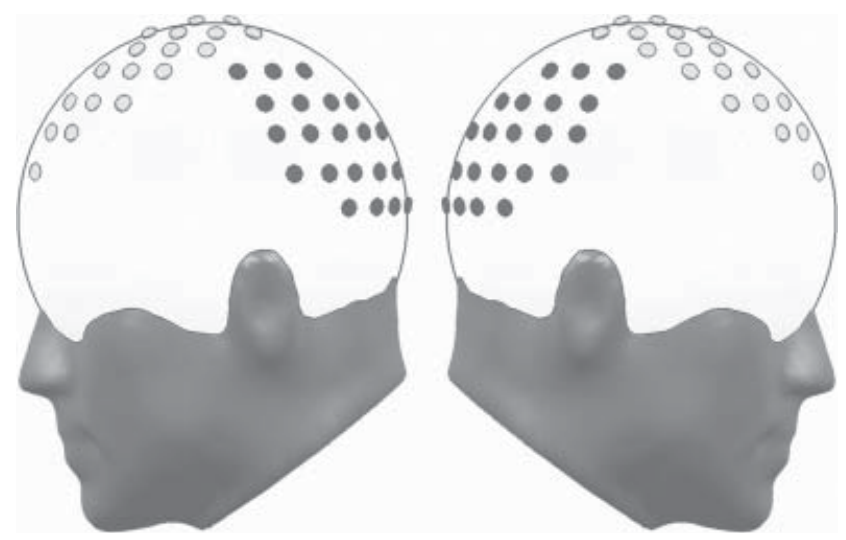

Figure 1. Schematic positions of the dipoles used for statistical analyses, displayed as left sided and right sided rendering of the spherical configuration. The dipoles forming the regions of interest (ROIs) are marked by black circles for posterior activity $160210 \mathrm{~ms}$ after stimulus onset, referred to as EPN, and by gray circles for anterior activity 80115 $\mathrm{ms}$, referred to as M100.

Mean ROI activity was submitted to ANOVAs containing the within subject factors Emotion (pleasant vs. neutral vs. un pleasant), Hemisphere (left ROI vs. right ROI), and, in separate analyses, Group (patients vs. comparison subject), Diagnosis (MDD vs. BPD vs. schizophrenia vs. DA) or ELS Group (low ELS vs. high ELS; see below). Nonspecific effects of psychopa thology were evaluated with Group (all patients vs. comparison subjects) $\times$ Emotion $\times$ Hemisphere (left and right occipito par ieto temporal ROI) ANOVAs. In addition, patients were com pared via Diagnosis (MDD, schizophrenia, DA, BPD) $\times$ Emotion $\times$ Hemisphere ANOVAs. These four group analyses were considered exploratory, given the small $N$ s per diagnostic subgroup. Significant main effects or interactions were explored with $t$ tests if not already clear from orthogonal trends. The Huynh Feldt epsilon correction accounted for possible viola tions of the homogeneity of covariance assumption (uncorrected degrees of freedom and epsilon corrected $p$ values are reported). The alpha level was set at .05 for each comparison.

ELS effects were first probed by Spearman rho $\left(r_{s}\right)$ and Pear son $(r)$ correlations including the entire sample. For simplicity in potential interactions with hemisphere, Group $\times$ Hemisphere ANOVAs were evaluated for ELS groups created by placing subjects with number of events above the mean $+2 S D$ of the comparison group into a high ELS group and subjects with number of events below the mean of the comparison group into a low ELS group.

Ratings of the valence and arousal properties of the stimuli were examined in an ANOVA with the Emotion factor com paring 25 pleasant, 25 neutral (randomly selected from the stim ulus set), and 25 unpleasant stimuli. Orthogonal trends captured valence (pleasant vs. unpleasant) as a linear trend and arousal (pleasant and unpleasant vs. neutral) as a quadratic trend. These trends reflected a priori hypotheses about critical dimensions of emotion (e.g., Lang, Bradley, \& Cuthbert, 1990), so tests of the orthogonal trends did not require a significant omnibus test.

\section{Results}

Stimulus Ratings and Stress History

Valence and arousal ratings (see Table 2) showed expected pat terns, primarily linear and quadratic, respectively (Bradley \& 
Table 2. Ratings of Valence and Arousal

\begin{tabular}{|c|c|c|c|c|c|c|}
\hline \multirow[b]{2}{*}{ Group: $N$} & \multicolumn{3}{|c|}{ Arousal $(M \pm S D)$} & \multicolumn{3}{|c|}{ Valence $(M \pm S D)$} \\
\hline & Pleasant & Neutral & Unpleasant & Pleasant & Neutral & Unpleasant \\
\hline Patients: 41 & $5.1 \pm 1.5$ & $3.2 \pm 1.4$ & $6.2 \pm 1.6$ & $6.6 \pm 1.2$ & $5.8 \pm 0.9$ & $2.1 \pm 0.8$ \\
\hline MDD: 12 & $4.9 \pm 1.7$ & $3.1 \pm 1.3$ & $6.5 \pm 1.8$ & $5.8 \pm 1.7$ & $5.8 \pm 0.7$ & $2.0 \pm 0.7$ \\
\hline Schizophrenia: 14 & $5.7 \pm 1.1$ & $3.7 \pm 1.2$ & $6.3 \pm 1.0$ & $6.8 \pm 1.0$ & $5.6 \pm 0.8$ & $2.2 \pm 0.6$ \\
\hline Drug addiction: 10 & $4.6 \pm 1.6$ & $2.7 \pm 1.5$ & $6.0 \pm 1.6$ & $7.2 \pm 0.8$ & $6.0 \pm 1.3$ & $2.1 \pm 0.6$ \\
\hline Borderline Personality Disorder: 5 & $4.8 \pm 1.4$ & $2.9 \pm 1.2$ & $5.7 \pm 2.7$ & $6.5 \pm 0.9$ & $5.5 \pm 0.5$ & $2.3 \pm 1.5$ \\
\hline Comparison subjects: 20 & $5.0 \pm 1.3$ & $3.2 \pm 1.3$ & $6.4 \pm 0.8$ & $6.5 \pm 0.8$ & $5.6 \pm 0.6$ & $2.2 \pm 0.8$ \\
\hline
\end{tabular}

Note: Ratings were given on a 9 point scale with higher values indicating higher arousal and more pleasant rating. Ratings were not available for 9 patients (7 MDD, 1 schizophrenia, 1 BPD).

Lang, 1994). In Group (patients, comparison subjects) $\times$ Emo tion analyses of valence and arousal ratings, pleasant pictures received the highest valence ratings and unpleasant the lowest, Emotion $F(2,116)=407.50, p<.001, \varepsilon=.93$. The linear trend, reflecting valence ratings for pleasant versus unpleasant pictures, contributed $89 \%$ of the Emotion variance, $F(1,58)=591.10$, $p<.001$, and the quadratic trend, reflecting pleasant and un pleasant versus neutral, contributed $11 \%$ of the Emotion vari ance, $F(1,58)=114.29, p<.001$. Unpleasant pictures were rated as more arousing than pleasant ones and both as more arousing than neutral ones, Emotion $F(2,118)=125.66, p<.001, \varepsilon=.90$. This was carried primarily by the quadratic trend, with $82 \%$ of the Emotion variance, $F(1,59)=191.37, p<.001$. The linear trend added $18 \%$ of the variance, $F(1,59)=46.76, p<.001 .^{4}$ Neither Group nor Group $\times$ Emotion effects approached sig nificance. In Diagnosis $\times$ Emotion analyses of valence and arousal ratings comparing the four patient subgroups, no effects involving diagnosis emerged. Thus, in ratings of stimuli, patients resembled comparison subjects.

Patients experienced a higher number of stressful events be fore onset of puberty (ELS) than did comparison subjects, $F(1,68)=20.32, p<.001$ (see Table 3). Among patients, ELS was higher in BPD and MDD than in schizophrenia or DA, Diag nosis $F(3,44)=8.88, p<.001$. Stimulus ratings were not associ ated with ELS or AS. The relationship between ELS and AS was explored before relating them to stimulus ratings. They were uncorrelated: Pearson $r(68)=.109, p=.37$; Spearman $r_{s}(68)=.178, p=.14$. Thus, high childhood stress load is not necessarily related to high adult stress load. Then, six hierarchical regressions were conducted with both early and adult stress pre dicting SAM ratings (separately for arousal and valence for each of pleasant, neutral, and unpleasant slides). None of the six om nibus tests (both life stress predictors in the model, each of the six ratings as the dependent variable) approached significance, and

${ }^{4}$ Although the IAPS stimuli were selected to match pleasant and un pleasant pictures on arousal value based on published norms (Center for the Study of Emotion and Attention, 2004), in the present sample un pleasant stimuli received slightly higher arousal ratings than did pleasant stimuli, raising the possibility of a confound of valence and arousal. However, arousal ratings for those two high arousal categories were much closer to each other than to arousal ratings for neutral stimuli: pleasant mean, $S D \quad 5.05,1.42$; neutral $3.19,1.31$; unpleasant $6.29,1.39$. Because the quadratic trend among these means represented more than 4 times the variance of the linear trend, it is clear that, as intended, arousal ratings largely reflected the arousal value of the stimuli and not their valence. Furthermore, because all of the findings for the EPN analysis involved quadratic (arousal) and not linear (valence) trends, the slight (though reliable) difference in arousal ratings for pleasant and unpleasant stimuli was not judged to be a significant confound for present purposes. in only 1 of 12 possible cases did either predictor add significant variance when entered second into the model. These results in dicate that, according to ratings, variations in stress history do not alter general emotional response and that ELS and AS are neither redundant measures nor confounds in relationships each may have with other variables, including MEG measures.

\section{EPN: ROI Activity at 160-210 ms}

Diagnosis and EPN. As shown in Figure 2, pleasant and un pleasant picture processing was associated with more posterior ROI activity than was neutral picture processing. The Emotion effect, $F(2,136)=6.33, p=.002, \varepsilon=1$, was carried by the qua dratic trend, $F(1,68)=12.17, p<.001$, explaining $95 \%$ of the Emotion variance. There was more activity in the right than in the left ROI, Hemisphere $F(1,68)=15.95, p<.001$. Patients showed a trend of less activity, Group $F(1,68)=3.31, p=.07$. No interaction approached significance.

Activity in visual processing areas differed by diagnosis: EPN was smaller in patients with MDD and BPD than in patients with schizophrenia, Diagnosis $F(3,46)=4.04, p=.01$, and Diagnosis $\times$ quadratic Emotion, $F(3,46)=2.77, p=.05$, explaining $89 \%$ of the Diagnosis $\times$ Emotion variance. Post hoc tests confirmed the differences between MDD and schizophrenia (Tukey HSD,

Table 3. Measures of Stress Load in the Different Subgroups

\begin{tabular}{|c|c|c|c|c|c|}
\hline Group: $N$ & $\begin{array}{c}\text { ELS } \\
M \pm S D \\
\text { (range) }\end{array}$ & $\begin{array}{c}\text { AS } \\
M \pm S D \\
\text { (range) }\end{array}$ & $\begin{array}{l}\text { High/low } \\
\text { ELS }\end{array}$ & $\begin{array}{l}\text { High/ } \\
\text { low AS }\end{array}$ & PTSD \\
\hline Patients: 50 & $\begin{array}{c}11.4 \pm 7.1 \\
\quad\left(\begin{array}{ll}0 & 32\end{array}\right)\end{array}$ & $\begin{array}{c}2.7 \pm 2.9 \\
(0 \quad 15)\end{array}$ & & & 13 \\
\hline MDD: 19 & $\begin{array}{c}13.9 \pm 6.2 \\
\quad(232)\end{array}$ & $\begin{array}{c}3.9 \pm 3.7 \\
(0 \quad 15)\end{array}$ & $14 / 1$ & $4 / 8$ & 9 \\
\hline Schizophrenia: 15 & $\begin{array}{c}6.9 \pm 5.3 \\
(019)\end{array}$ & $\begin{array}{c}1.8 \pm 1.3 \\
\left(\begin{array}{ll}0 & 4\end{array}\right)\end{array}$ & $5 / 6$ & $0 / 11$ & 0 \\
\hline Drug addiction: 10 & $\begin{array}{c}8.7 \pm 4.0 \\
(417)\end{array}$ & $\begin{array}{c}2.0 \pm 2.6 \\
\left(\begin{array}{ll}0 & 8\end{array}\right)\end{array}$ & $4 / 1$ & $1 / 4$ & 0 \\
\hline $\begin{array}{l}\text { Borderline } \\
\text { Personality }\end{array}$ & $\begin{aligned} 19.3 & \pm 8.5 \\
(6 & 28)\end{aligned}$ & $\begin{array}{c}2.2 \pm 2.8 \\
\left(\begin{array}{ll}0 & 7\end{array}\right)\end{array}$ & $5 / 0$ & $1 / 1$ & 4 \\
\hline $\begin{array}{l}\text { Disorder: } 6 \\
\text { Comparison } \\
\text { subjects: } 20\end{array}$ & $\begin{array}{c}4.1 \pm 2.6 \\
\left(\begin{array}{ll}0 & 10)\end{array}\right)\end{array}$ & $\begin{array}{c}2.6 \pm 2.7 \\
(0 \quad 10)\end{array}$ & $1 / 12$ & $2 / 12$ & 0 \\
\hline
\end{tabular}

Note: ELS early life stress: number of stressful events reported for the time before the individual onset of puberty; AS adult stress: number of stressful events reported after age 18. Early Trauma Inventory scores were not available for 2 patients. PTSD: number of subjects with co morbid diagnosis of posttraumatic stress disorder based on the PDS in terview. Note that the number of high and low ELS subjects does not add up to the number of studies subjects, as subjects at the extreme of the number of event dimension were assigned to the subgroups. 


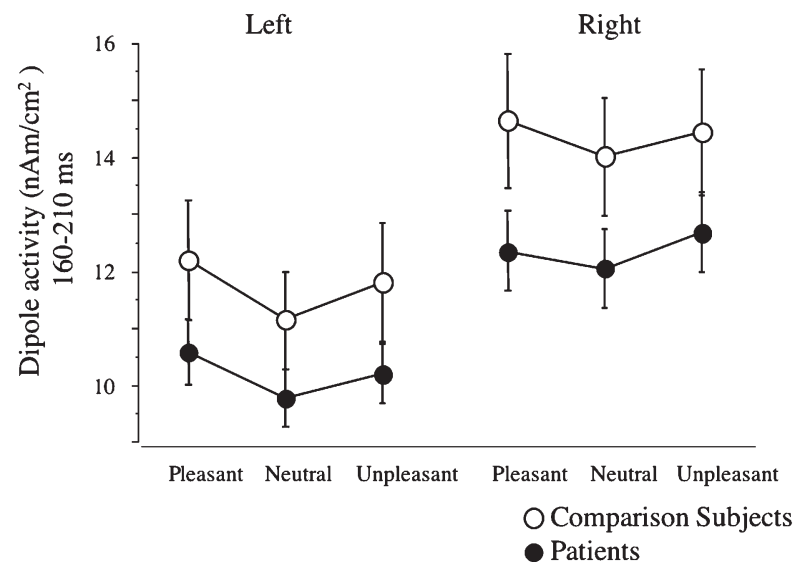

Figure 2. Modulation of posterior dipole activity at $160210 \mathrm{~ms}$ by group (patients: filled black circles, comparison subjects: open circles), emotion (abscissa), and hemisphere. Ordinate: Dipole activity expressed as minimum norm estimates, mean \pm standard error in $\mathrm{nAm} / \mathrm{cm}^{2}$.

$p<.05)$ and between BPD and schizophrenia $(p<.05)$; other pairs of groups did not differ. When comparing the different diagnostic subgroups with comparison subjects, right hemi spheric EPN differences were verified between MDD patients and comparison subjects, $t(37)=2.21, p=.03$, and BPD and comparison subjects, $t(24)=2.77, p=.01$, but not between schizophrenia or DA patients and comparison subjects.

Pursuing the Group $\times$ quadratic Emotion interaction, visual inspection suggested that MDD and BPD patients did not show an arousal effect, compared to schizophrenia and DA patients. MDD and BPD patients might well be similar in emotional pro cessing abnormalities, given their frequently considerable co morbidity. Indeed, the Emotion main effect was not significant for the combined group of MDD and BPD patients, $F(2,48)<1$, quadratic Emotion $F(1,24)<1$, whereas it was for patients with schizophrenia and DA, $F(2,48)=8.92, p<.001, \varepsilon=.73$, qua dratic Emotion $F(1,24)=10.95, p=.003$. This suggests that, in addition to overall EPN suppression, affective modulation is impaired in patients with MDD and BPD.

ELS and EPN. As reported above, patients experienced more ELS than did comparison subjects, and among patients, ELS was higher in BPD and MDD than in schizophrenia or DA. As a consequence, it is difficult to distinguish the contributions of specific diagnosis versus ELS to electromagnetic responses. As shown in Table 4 and Figure 3, ELS score correlated negatively

Table 4. Spearman Correlations between Stress Load and EPN Amplitude

\begin{tabular}{|c|c|c|c|c|c|c|}
\hline & \multicolumn{3}{|c|}{ EPN right hemisphere } & \multicolumn{3}{|c|}{ EPN left hemisphere } \\
\hline & Pleasant & Neutral & Unpleasant & Pleasant & Neutral & Unpleasant \\
\hline ELS & $-.36^{* * *}$ & $-.38 * *$ & $-.40^{* * *}$ & $-.35 * *$ & $-.24 *$ & $-.28 *$ \\
\hline AS & -.10 & -.09 & -.08 & -.14 & -.06 & -.08 \\
\hline
\end{tabular}

Note: $N$ 70. ELS early life stress: number of stressful events reported for the time before the individual onset of puberty; AS adult stress: number of stressful events reported after age 18 .

$* p<.05$, two tailed;

$* * *<<.01$ level, two tailed.

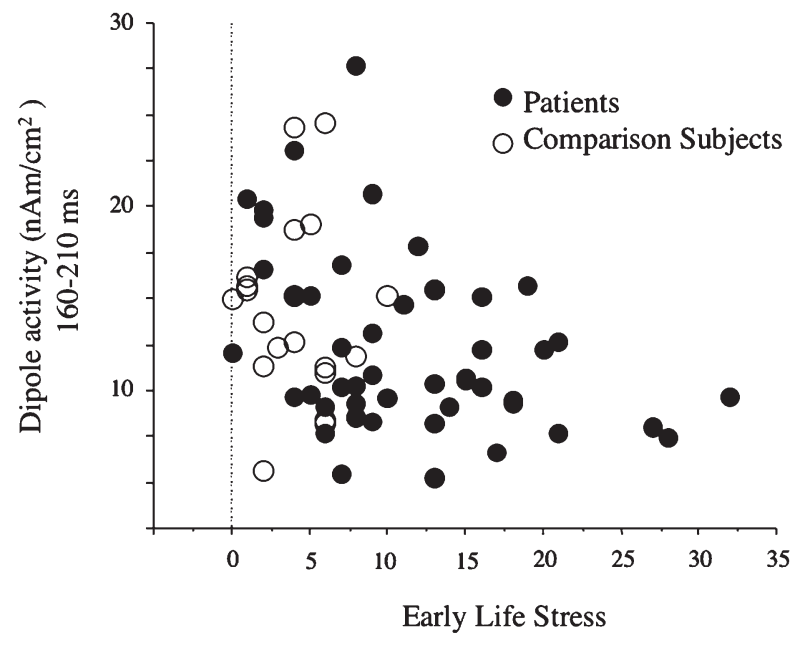

Figure 3. Relationship between stress load before puberty (ELS, abscissa: number of experienced events between birth and individual onset of puberty) and right hemisphere EPN averaged across all three levels of emotion for patients (filled black circles) and comparison subjects (open circles). Ordinate: Dipole activity expressed as minimum norm estimates, mean \pm standard error in $\mathrm{nAm} / \mathrm{cm}^{2}$.

with EPN ROI activity elicited by pleasant, neutral, and un pleasant pictures, somewhat more pronounced for right than left ROIs. In contrast to the consistent relationship for ELS, AS did not differentiate patients and comparison subjects $(F<1$; Table 3 ) or diagnostic subgroups, $p=.12$. None of the six correlations between AS and EPN approached significance (Table 4).

The ELS/EPN relationship was examined further by com paring high and low ELS groups (high ELS: $N=26$, including 25 patients; low ELS: $N=20,8$ patients). ${ }^{5}$ High ELS subjects had less active visual regions, $F(1,44)=13.14, p<.001$. How ever, the affective modulation of EPN, $F(2,88)=5.55, p=.005$, $\varepsilon=1.00$, was not influenced by ELS (ELS Group $\times$ Emotion interaction, $p=.14)$. Activity was right lateralized, $F(1,44)=$ $18.58, p<.001$, with an ELS Group $\times$ Hemisphere interaction, $F(1,44)=4.41, p=.04$, indicating less EPN lateralization for the high ELS group, Hemisphere $F(1,25)=4.58, p=.04$, than in the low ELS group, $F(1,19)=12.05, p=.002$, and more differenti ation between ELS groups in the right, $F(1,44)=15.12$, $p<.001)$, than in the left hemisphere, $F(1,44)=3.62, p=.06$ (see Figure 4). ELS was generally related to EPN suppression, particularly in the right hemisphere (see Table 4 and Figure 3).

PTSD and EPN. There was overlap between comorbid PTSD (determined by PDS) and ELS in that the number of patients with comorbid PTSD was higher in the diagnostic sub groups with high ELS (MDD and BPD; see Table 3). An issue is the extent to which ELS effects on EPN were redundant with possible effects of PTSD on EPN. First, ELS score and PTSD (meets criteria for PTSD diagnosis or not) were indeed corre lated: Pearson $r(68)=.428, p<.001$; Spearman $r_{s}(68)=.452$,

${ }^{5}$ High and low ELS subgroups did not differ with respect to the number of trials that entered analyses $(p>.2)$. As indicated in Table 3 , the assignment of patients to the high ELS and low ELS groups differed by diagnosis, $\chi^{2}$ (3) $10.2, p<.02$ (including the comparison group: $\left.\chi^{2}[4] 26.7, p<.001\right)$. This was not the case for assignment to sub groups with high and low number of stressful events in adulthood, AS: $\chi^{2}$ (3) $5.21, p \quad .16$; including comparison subjects, $\chi^{2}(4) \quad 5.81, p>.2$. 


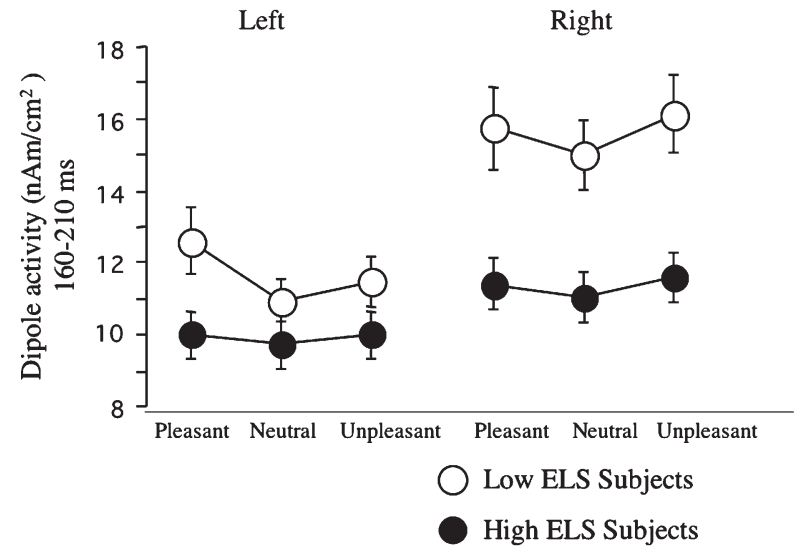

Figure 4. Modulation of dipole activity at $160210 \mathrm{~ms}$ (EPN) by ELS group, emotion, and hemisphere. Filled black circles: subjects with number of stressful events more than $2 S D$ above the mean of the comparison group. Open circles: subjects with number of stressful events below the mean of the comparison group. Ordinate: Dipole activity expressed as minimum norm estimates, mean \pm standard error in $\mathrm{nAm} / \mathrm{cm}^{2}$.

$p<.001$. However, hierarchical regression showed that high ELS was associated with EPN suppression separate from any contri bution from or redundancy with PTSD. For example, a regres sion employing ELS score and PTSD status as predictors accounted for $14 \%$ of the variance in right hemisphere EPN score for unpleasant stimuli, $F(2,67)=5.48, p=.006$. Added second, PTSD diagnosis did not add variance, $p=.30$, to that accounted for by EPN, whereas EPN did add unique variance, $p=.02$. Thus, ELS related to EPN suppression above and be yond the role of PTSD diagnosis.

$B D I$ and $E P N$. As a final question about the specificity of ELS/EPN effects, mood symptom severity might mediate this relationship. ELS and BDI score were correlated: Pearson $r(68)=.478, p<.001$; Spearman $r_{s}(68)=.510, p<.001$. Hierar chical regression showed that high ELS was associated with EPN suppression separate from any contribution from or redundancy with BDI. For example, a regression employing ELS score and BDI score as predictors accounted for $22 \%$ of the variance in right hemisphere EPN score for unpleasant slides, $F(2,67)=$ $8.88, p<.001$. Added second, both ELS, $p<.001$, and BDI, $p=.007$, added unique variance. Thus, ELS related to EPN suppression above and beyond the role of BDI.

\section{M100: ROI Activity at 80-115 ms}

It is possible that posterior brain activity in the $160210 \mathrm{~ms}$ time window reflects a more general phenomenon observable earlier in the processing stream. To address this issue, activity was analyzed in left and right anterior ROI in an $80115 \mathrm{~ms}$ time window. No evidence for Group $(p=.4)$ or Emotion $(p=.5)$ main effects emerged. A Group $\times$ Emotion interaction, $F(2,136)=4.14$, $p=.02$, was carried by a linear trend, $F(1,68)=5.39, p=.02$, explaining $64 \%$ of the variance. Inspection revealed no signifi cant Emotion effect in patients, whereas in comparison subjects Emotion approached significance, $F(2,38)=3.12, p=.06$, $\varepsilon=.85$. The weakness of these effects and the absence of a Group $\times$ quadratic Emotion effect indicate that the Group $\times$ quadratic Emotion findings in the later (EPN) time window are not simply left over from early processing. The distinct effects support the specificity of findings in the EPN time window.

\section{Discussion}

The present study explored whether early life stress (ELS) influ ences brain activity during the processing of emotionally salient material and whether this influence is similar in normal subjects and in patients with psychiatric disorders. Results confirmed the reported modulation of electromagnetic activation in posterior regions $160210 \mathrm{~ms}$ after stimulus onset (apparently comparable to the early posterior negativity in EEG and therefore labeled EPN in the present study) by visual stimuli as a function of emotional content (Lang et al., 1998b; Schupp et al., 2003, 2004). Using a slightly earlier time window (120 $170 \mathrm{~ms}$ ) Peyk et al. (2008) described similar modulation of posterior brain activity by stimulus valence and related it to emotional attention capture in the visual processing stream. In contrast, the present study con firmed anterior modulation around $100 \mathrm{~ms}$ only as a trend in healthy subjects.

As a group, present psychiatric inpatients showed normal EPN modulation as a function of stimulus arousal value, indi cating that the initial cortical analysis or "tagging" (Halgren \& Marinkovic, 1995) of cues as behaviorally relevant is intact in such patients. However, both ELS and psychiatric disorder were associated with altered EPN response to emotional stimuli. Pa tients with MDD and BPD, in particular, exhibited reduced EPN and less affective modulation of EPN than did patients with schizophrenia or healthy subjects. Cortical correlates of abnor mal affective processing in psychiatric patients have been found in fMRI, EEG, and MEG studies. Schizophrenia primarily shows reduced activation by emotionally salient stimuli and re duced modulation of brain activity as a function of stimulus valence (for a review, see Rockstroh et al., 2006). In MDD, Moratti, Rubio, Campo, Keil, and Ortiz (2008) found weak arousal modulation of right temporal MEG activity by IAPS pictures. Yee and Miller (1988) observed an affective processing shutdown when dysthymic subjects anticipated such stimuli. Ev idence from fMRI suggests reduced activity in hippocampus and insula to unpleasant pictures and reduced ACC and amygdala activity to pleasant pictures in MDD (Lee et al., 2007). These examples suggest dampened rather than enhanced emotional ac tivation in some types of psychopathology. Whereas the EPN has not previously been specifically addressed in these psychiatric groups, the slow wave component of the ERP in the latency range of $300400 \mathrm{~ms}$ confirmed reduced sustained activity during the processing of faces (Deldin, Keller, Gergen, \& Miller, 2000; see also Kayser, Bruder, Tenke, Stewart, \& Quitkin, 2000) or positive words (Shestyuk, Deldin, Brand, \& Deveney, 2005), which the authors proposed as a contributing factor for cognitive deficits in MDD (see also Deveney \& Deldin, 2004).

Abnormal cortical responses to affective stimuli do not nec essarily predict abnormal behavioral responses: Discrepancies between reduced affective modulation of early cortical responses but normal ratings of stimulus valence have been reported before (Rockstroh et al., 2006). In schizophrenia patients it was related to compensatory functions, which allow normal processing of emotional stimuli whenever they are presented without time constraints or workload, despite indications of abnormal auto matic processing. This explanation might hold as well for the present psychiatric sample. That measures of symptom severity (BPRS, BDI) did not vary with EPN whereas diagnosis and ELS 
did suggests that present results reflect trait characteristics rather than current status of severe psychopathology.

High early life stress was also associated with EPN suppres sion, although specific additive versus interactive effects of ELS and psychiatric disorder cannot be fully distinguished in the present study. Indeed, because ELS contributes to psychiatric disorder and third variables promoting psychiatric disorder may also prompt ELS, a thorough differentiation, if possible, would require a much larger sample assessed for additional risk factors (e.g., genetic markers and environmental contributors). The present high ELS group comprised more MDD and BPD pa tients than schizophrenia and drug addiction patients and the low ELS group more patients with schizophrenia than patients with other diagnoses. This ELS/diagnosis association might be con sidered a confound, hampering identification of specific ELS effects. However, isolated main effects are less likely than Gene $\times$ Environment interactions, given current thinking in the psy chopathology literature (e.g., Kendler, 2005; Turkheimer, 2000): Most of the variance is likely to be in the interactions, not in gene or environment main effects. The present study identified a sub group of individuals in which ELS and other risk factors may have promoted a pattern of depressive and borderline symptoms and potentially also comorbid PTSD together with altered affec tive processing. Whereas both factors, ELS and affective mod ulation of brain responses, have not been examined within the same design, findings of high ELS in MDD (Heim \& Nemeroff, 2002; Heim et al., 2004; Moratti et al., 2008) and dampened cortical responding in MDD (as described above) support the existence of such a subgroup. Moreover, comorbidity has been reported for PTSD and MDD (e.g., Breslau, 2002; Franklin \& Zimmerman, 2001) and for BPD and PTSD (e.g., Clarke, Rizvi, \& Resick, 2008; Heffernan \& Cloitre, 2000). From this comorbidity, one might assume that the present relationship between ELS and EPN reflects the relationship between PTSD and EPN irrespec tive of stress load. However, the few studies that have examined affect related brain activation in patients with PTSD reported cortical hyperactivity (e.g., Attias, Bleich, Furman, \& Zinger, 1996; Ehlers et al., 2006; Felmingham, Bryant, \& Gordon, 2003) rather than reduced activation in the EPN latency range. Im portantly, present analyses provided evidence of specificity for the ELS/EPN relationship that it is not merely a function of redundancy with adult stress, PTSD diagnosis, affective symp toms (BDI score), or responses earlier in the trial. Studies with larger psychiatric samples are needed to pursue the relationship between ELS and EPN and the role of specific types of psycho pathology in that relationship.

Altered EPN has been related to brain circuits processing unpleasant or anxiety provoking stimuli (LeDoux, 2000). In healthy subjects, task irrelevant, high arousing pictures reduced N100 and EPN to subsequent task relevant stimuli (Ihssen, Heim, \& Keil, 2007), indicating arousal dependent interference. It is tempting to speculate that severe stress load many years earlier can exert similar effects. In animal experiments, altered brain activity has been related to stress induced helplessness (Cabib \& Puglisi Allegra, 1996; Porsolt et al., 1978), which is also relevant for altered brain activation in individuals with ELS and MDD or BPD (Lang et al., 2007; Morley et al., 2003). Stress induced hyperactivity of the stress response system (Char mandari et al., 2003) or the defense system (Lang, Davis, \& Öhman, 2000) during sensitive developmental periods has been assumed to mediate changes in brain structure and function in volved in affect regulation, which may be reflected in dampened cortical responses to emotional stimuli.

Methodological limitations of the present study may be noted. The null hypothesis of uniform ELS effects across diag nostic subgroups was rejected, but this involved splitting the pa tient sample $(n=50)$ into diagnostic subgroups of unequal size, prompting power differentials in some follow up analyses. In particular, results for BPD warrant verification in a larger sam ple. Moreover, a gender imbalance in diagnostic prevalence is well known, with a higher proportion of women diagnosed with MDD or BPD and a higher proportion of men diagnosed with schizophrenia or drug abuse. The present sample represented this asymmetry (association between gender and diagnostic subtype, $\chi^{2}[3]=12.25, p=.007$ ). Nevertheless, when the Group (patients vs. comparison subjects $) \times$ Emotion $\times$ Hemisphere ANOVAs for EPN were repeated with Gender added as a factor, no main effect or interaction involving Gender was obtained. Thus, there was no interpretive confound with gender here. Several left handed or ambidextrous subjects were included, to preserve $N \mathrm{~s}$ (especially in the diagnostic subgroups) and to avoid misrepre senting handedness in clinical samples. However, the main find ings reported here did not depend on hemisphere interactions, so inclusion of such subjects does not appear to be a problem. Fi nally, clinical routine at the local Center for Psychiatry (which primarily treats chronic inpatients from the region) did not allow examination of nonmedicated patients or subgroups of patients with monotherapy.

In sum, exploring the relationship between ELS, psychiatric disorder, and cortical affective processing suggests that ELS in teracts with type of psychiatric disorder in its effects on cortical processing. These findings suggest a dynamic relationship between vulnerability to psychopathology and an ELS induced or medi ated risk for aggravation of psychopathology. Larger samples se lected for high and low ELS, unconfounded with diagnostic subgroup, would be valuable to substantiate this interaction.

\section{REFERENCES}

Ackenheil, M., Stotz Ingenlath, G., Dietz Bauer, R., \& Vossen, A. (1998). Mini International Neuropsychiatric Interview, German Ver sion. Munich, Germany: Department of Psychiatry, University of Munich.

Attias, J., Bleich, A., Furman, V., \& Zinger, Y. (1996). Event related potentials in post traumatic stress disorder of combat origin. Biolog ical Psychiatry, 40, 373381.

Baillet, S., Mosher, J. C., \& Leahy, R. M. (2001). Electromagnetic brain mapping. IEEE Signal Processing Magazine, 18, 1430.

Borgelt, J., Odenwald, M., Ruf, M., Elbert, T., \& Kissler, J. (2009). Rapid orbito frontal cortex activation to aversive pictures in Posttraumatic Stress Disorder. Manuscript submitted for publication.
Bradley, M. M., Codispoti, M., Cuthbert, B. N., \& Lang, P. J. (2001). Emotion and motivation I: Defensive and appetitive reactions in pic ture processing. Emotion, 1, 276299.

Bradley, M. M., \& Lang, P. J. (1994). Measuring emotion: The Self Assessment Manikin and the semantic differential. Journal of Behav ioral Therapy and Experimental Psychiatry, 25, 4549.

Bradley, M. M., Sabatinelli, D., Lang, P. J., Fitzsimmons, J. R., King, W., \& Desai, P. (2003). Activation of the visual cortex in motivated attention. Behaviorial Neuroscience, 117, 369380.

Bremner, J. D. (2002). Does stress damage the brain? New York: Norton.

Bremner, J. D., Bolus, R., \& Mayer, E. A. (2007). Psychometric prop erties of the Early Trauma Inventory Self Report. Journal of Nervous and Mental Disease, 195, 211218. 
Bremner, J. D., Vermetten, E., \& Mazure, C. M. (2000). Development and preliminary psychometric properties of an instrument for the measurement of childhood trauma: The early trauma inventory. De pression and Anxiety, 12, 112.

Breslau, N. (2002). Epidemiologic studies of trauma, posttraumatic stress disorder, and other psychiatric disorders. Canadian Journal of Psy chiatry, 47, 923929.

Cabib, S., \& Puglisi Allegra, S. (1996). Stress, depression and the me solimbic dopamine system. Psychopharmacology, 128, 331342.

Center for the Study of Emotion and Attention. (2004). The International Affective Picture System: Digitized photographs. Gainesville, FL: Center for Research in Psychophysiology, University of Florida.

Charmandari, E., Kino, T., \& Souvatzoglou, E. (2003). Pediatric stress: Hormonal mediators and human development. Hormone Research, $59,161179$.

Clarke, S. B., Rizvi, S. L., \& Resick, P. A. (2008). Borderline personality characteristics and treatment outcome in cognitive behavioral treatments for PTSD in female rape victims. Behavior Therapy, 39, 7278.

Cohen, R. A., Paul, R. H., Stroud, R. H., Gunstad, J., Hitsman, B. L., McCaffrey, J., et al. (2006). Early life stress and adult emotional experiences: An international perspective. International Journal of Psychiatry in Medicine, 36, 3552.

De Bellis, M. D. (2002). Developmental traumatology: Contributory mechanism for alcohol substance use disorders. Psychoneuroendocri nology, 27, 155170.

Deldin, P. J., Keller, J., Gergen, J. A., \& Miller, G. A. (2000). Right posterior face processing anomaly in depression. Journal of Abnormal Psychology, 109, 116121.

Deveney, C. M., \& Deldin, P. J. (2004). Memory of faces: A slow wave ERP study of major depression. Emotion, 4, 295304.

Ehlers, C. L., Hurst, S., Phillips, E., Gilder, D. A., Dixon, M., Gross, A., et al. (2006). Electrophysiological responses to affective stimuli in American Indians experiencing trauma with and without PTSD. An nals of the New York Academy of Sciences, 1071, 125136.

Felmingham, K. L., Bryant, R. A., \& Gordon, E. (2003). Processing angry and neutral faces in post traumatic stress disorder: An event related potentials study. NeuroReport, 14, 777780.

Foa, E. B. (1995). Posttraumatic stress diagnostic scale (PDS). Minne apolis, MN: National Computer Systems.

Franklin, C. L., \& Zimmerman, M. (2001). Posttraumatic stress disorder and major depressive disorder: Investigating the role of overlapping symptoms in diagnostic comorbidity. Journal of Nervous and Mental Disease, 189, 548551.

Garieballa, S., Schauer, M., Neuner, F., Saleptsi, E., Elbert, T., Hoffm ann, K., et al. (2006). Traumatic events, PTSD, and psychiatric co morbidity in forensic patients assessed by questionnaire and diagnostic interview. BMC Clinical Practice and Epidemiology in Mental Health, 2, 17.

Goodman, M., New, A., \& Siever, L. (2004). Trauma, genes, and the neurobiology of personality disorders. Annals of the New York Acad emy of Sciences, 1032, 104116.

Halgren, E., \& Marinkovic, K. (1995). Neurophysiological networks in tegrating human emotions. In M. S. Gazzaniga (Ed.), The cognitive neurosciences (pp. 1137 1151). Cambridge, MA: MIT Press.

Hamalainen, M., \& Ilmoniemi, R. (1994). Interpreting magnetic fields of the brain: Estimates of current distribution. Technical Report TKK F A559. Helsinki: Helsinki University of Technology.

Hauk, O. (2004). Keep it simple: A case for using classical minimum norm estimation in the analysis of EEG and MEG data. NeuroImage, 21,16121621

Hauk, O., Keil, A., Elbert, T., \& Muller, M. M. (2002). Comparison of data transformation procedures to enhance topographical accuracy in time series analysis of the human EEG. Journal of Neuroscientific Methods, 113, 111122.

Hautzinger, M., Bailer, M., Worall, H., \& Keller, F. (1994). Beck De pressions Inventar (BDI). Bern, Switzerland: Huber.

Heffernan, K., \& Cloitre, M. (2000). A comparison of posttraumatic stress disorder with and without borderline personality disorder among women with a history of childhood sexual abuse: Etiological and clinical characteristics. Journal of Nervous and Mental Disease, $188,589595$.

Heim, C. (2000). Deutsche Version des Early Trauma Inventory: Inventar zur Erfassung früher traumatischer Lebensereignisse (IFTL) [German version of the Early Trauma Inventory: Inventory for the assessment of early traumatic life events]. Unpublished manuscript, Emory Uni versity School of Medicine.

Heim, C., \& Nemeroff, C. B. (2002). Neurobiology of early life stress: Clinical studies. Seminars in Clinical Neuropsychiatry, 7, 147159.

Heim, C., Plotsky, P., \& Nemeroff, C. B. (2004). The importance of studying the contributions of early adverse experience to neurobiol ogical findings in depression. Neuropsychopharmacology, 29, 641 648.

Ihssen, N., Heim, S, \& Keil, A. (2007). The costs of emotional attention: Affective processing inhibits subsequent lexico semantic analysis. Journal of Cognitive Neuroscience, 19, 19321949.

Junghofer, M., Bradley, M. M., Elbert, T. R., \& Lang, P. J. (2001). Fleeting images: A new look at early emotion discrimination. Psy chophysiology, 38, 175178.

Junghofer, M., \& Peyk, P. (2004). Analyse und Visualisierung von Hi rnstrom und Hirnmagnetfeld Messungen [Analysis and visualization of measurements of electrical potentials and electromagnetic fields]. MATLAB Select, 2, 2428.

Junghofer, M., Sabatinelli, D., Bradley, M. M., Schupp, H. T., Elbert, T. R., \& Lang, P. J. (2006). Fleeting images: Rapid affect discrimination in the visual cortex. NeuroReport, 17, 225229.

Junghofer, M., Schauer, M., Neuner, F., Odenwald, M., Rockstroh, B., \& Elbert, T. (2003). Enhanced fear network in torture survivors ac tivated by RSVP of aversive material can be monitored by magnetic source imaging. Psychophysiology, 40(Suppl. 3).

Junghofer, M., Schupp, H. T., Stark, R., \& Vaitl, D. (2005). Neuro imaging of emotion: Empirical effects of proportional global signal scaling in fMRI data analysis. NeuroImage, 25, 520526.

Kayser, J., Bruder, G., Tenke, C. E., Stewart, J. E., \& Quitkin, F. M. (2000). Event related potentials (ERPs) to hemifield presentations of emotional stimuli: Differences between depressed patients and healthy adults in P3 amplitude and asymmetry. International Jour nal of Psychophysiology, 36, 211236.

Kendler, K. S. (2005). "A gene for...": The nature of gene action in psychiatric disorders. American Journal of Psychiatry, 162, 1243 1252

Kendler, K. S., Kuhn, J. W., Vittum, J., Prescott, C. A., \& Riley, B. (2005). The interaction of stressful life events and a serotonin transporter polymorphism in the prediction of episodes of major de pression: A replication. Archives of General Psychiatry, 62, 529535.

Lang, P. J., Bradley, M. M., \& Cuthbert, B. N. (1990). Emotion, at tention, and the startle reflex. Psychological Review, 97, 377395.

Lang, P. J., Bradley, M. M., \& Cuthbert, B. N. (1997). Motivated at tention: Affect, activation, and action. In P. J. Lang, R. F. Simons, \& M. Balaban (Eds.), Attention and emotion: Sensory and motivational processes (pp. 97 135). Mahwah, NJ: Erlbaum.

Lang, P. J., Bradley, M. M., \& Cuthbert, B. N. (1998a). Emotion and motivation: Measuring emotional perception. Journal of Clinical Neurophysiology, 15, 397408.

Lang, P. J., Bradley, M. M., \& Cuthbert, B. N. (1998b). Emotion, motivation, and anxiety: Brain mechanisms and psychophysiology. Biological Psychiatry, 44, 12481263.

Lang, P. J., Bradley, M. M., \& Cuthbert, B. N. (1999). International Affective Picture System (IAPS): Instruction manual and affective ratings. Technical report A 4. Gainesville, FL: Center for Research in Psychophysiology, University of Florida.

Lang, P., Davis, M., \& Ohman, A. (2000). Fear and anxiety: Animal models and human cognitive psychophysiology. Journal of Affective Disorders, 61, 137159.

Lang, P. J., McTeague, L., \& Cuthbert, B. N. (2007). Fear, anxiety, depression, and the anxiety disorder spectrum: A psychophysiological analysis. In T. Baker, R. Bootzin, \& T. Treat (Eds.), Psychological clinical science: Recent advances in theory and practice (pp. 167 195). Hillsdale, NJ: Erlbaum.

LeDoux, J. E. (2000). Emotion circuits in the brain. Annual Review of Neuroscience, 23, 155184.

Lee, B. T., Seong, W. C., Hyung, S. K., Lee, B. C., Choi, I. G., Lyoo, I. K., et al. (2007). The neural substrates of affective processing toward positive and negative affective pictures in patients with major depres sive disorder. Progress in Neuropsychopharmacology and Biological Psychiatry, 31, 14871492.

Lukoff, D., Liberman, R. P., \& Nuechterlein, K. H. (1986). Symp tom monitoring in the rehabilitation of schizophrenic patients. Schizophrenia Bulletin, 12, 578 602. Modification of: Overall, E., \& 
Gorham, D.R. (1962). The brief psychiatric rating scale. Psycholog ical Reports, 10, 799812.

Mayberg, H. (2003). Positron emission tomography imaging in depres sion: A neural systems perspective. Neuroimaging Clinics of North America, 13, 805815 .

Maynard, T. M., Sikich, L., Lieberman, J. A., \& LaMantia, A. S. (2001). Neural development, cell cell signaling, and the 'two hit' hypothesis of schizophrenia. Schizophrenia Bulletin, 27, 457476.

McEwen, B. S. (2003). Early life influences on life long patterns of be havior and health. Mental Retardation and Developmental Disability Research Review, 9, 149154.

Moratti, S., Rubio, G., Campo, P., Keil, A., \& Ortiz, T. (2008). Hy pofunction of right temporoparietal cortex during emotional arousal in depression. Archives of General Psychiatry, 65, 110.

Morley, S., Darnaudery, M., Koehl, M., Casolini, P., Van Reeth, O., \& Maccarim, S. (2003). Prenatal stress in rats predicts immobility be havior in the forced swim test. Effects of a chronic treatment with tianeptine. Brain Research, 989, 246251.

Nemeroff, C. B. (2004). Neurobiological consequences of childhood trauma. Journal of Clinical Psychiatry, 65, 1828.

Oldfield, R. C. (1970). Assessment and analysis of handedness: The Ed inburgh inventory. Neuropsychologia, 9, 97113.

Paivio, S. C., \& Cramer, K. M. (2004). Factor structure and reliability of the Childhood Trauma Questionnaire in a Canadian undergraduate student sample. Child Abuse and Neglect, 28, 889904.

Peyk, P., Schupp, H. T., \& Elbert, T. (2008). Emotion processing in the visual brain: A MEG analysis. Brain Topography, 20, 205 215

Pole, N., Neylan, T. C., Otte, C., Metzler, T. J., Best, S. R., Henn Haase, C., et al. (2007). Association between childhood trauma and emotion modulated psychophysiological responses to startling sounds: A pilot study of police cadets. Journal of Abnormal Psychology, 116, 352361.

Porsolt, R. D., Bertin, A., \& Jalfre, M. (1978). "Behavioural despair" in rats and mice: Strain differences and the effects of imipramine. Eu ropean Journal of Pharmacology, 51, 291297.

Rockstroh, B., Junghofer, M., Elbert, T., Buodo, G., \& Miller, G. A. (2006). Electromagnetic brain activity avoked by affective stimuli in schizophrenia. Psychophysiology, 43, 431439.
Sabatinelli, D., Bradley, M. M., Fitzsimmons, J. R., \& Lang, P. J. (2005). Parallel amygdala and inferotemporal activation reflect emotional intensity and fear relevance. NeuroImage, 24, 12651270.

Scheller Gilkey, G., Moynes, K., Cooper, I., Kant, C., \& Miller, A. H. (2004). Early life stress and PTSD symptoms in patients with comor bid schizophrenia and substance abuse. Schizophrenia Research, 69, 167174.

Schupp, H. T., Junghofer, M., Weike, A. I., \& Hamm, A. O. (2003). Emotional facilitation of sensory processing in the visual cortex. Psy chological Science, 14, 713.

Schupp, H. T., Junghofer, M., Weike, A. I., \& Hamm, A. O. (2004). The selective processing of briefly presented affective pictures: An ERP study. Psychophysiology, 41, 441449.

Shestyuk, A. Y., Deldin, P. J., Brand, J. E., \& Deveney, C. M. (2005). Reduced sustained brain activity during processing of positive emo tional stimuli in major depression. Biological Psychiatry, 57, 1089 1096.

Sinha, R. (2005). Stress and drug abuse. In T. Steckler, N. Kalin, \& J. Reul (Eds.), Handbook of stress and the brain (Vol. 15, pp. 333 347). Amsterdam: Elsevier.

Smith, N. K., Cacioppo, J. T., Larsen, J. T., \& Chartrand, T. L. (2003). May I have your attention, please: Electrocortical responses to pos itive and negative stimuli. Neuropsychologia, 41, 171183.

Thompson, J., Pogue Geile, M., \& Grace, A. (2004). Developmental pathology, dopamine and stress: A model for the age of onset of schizophrenia symptoms. Schizophrenia Bulletin, 30, 875900.

Turkheimer, E. (2000). Three laws of behavior genetics and what they mean. Current Directions in Psychological Science, 9, 160164.

Van der Kolk, B. A., Roth, S., Pelcovitz, D., Sunday, S., \& Spinazzola, J. (2005). Disorders of extreme stress: The empirical foundation of a complex adaptation to trauma. Journal of Traumatic Stress, 18, 389 399.

Yee, C. M., \& Miller, G. A. (1988). Emotional information processing: Modulation of fear in normal and dysthymic subjects. Journal of Abnormal Psychology, 97, 5463. 